American Journal of Applied Sciences 9 (2): 238-239, 2012

ISSN 1546-9239

(C) 2012 Science Publications

\title{
Flow Based Algorithm
}

\author{
Karpagam, T., V.J. Sudhakar, N. Arunsankar and M. Dhanasekaran \\ Department of Management Science, \\ Adhiyamaan College of Engineering, Hosur, Tamilnadu 602 105, India
}

\begin{abstract}
Problem statement: Network topology design problems find application in several real life scenario. Approach: Most designs in the past either optimize for a single criterion like shortest or cost minimization or maximum flow. Results: This study discussed about solving a multi objective network topology design problem for a realistic traffic model specifically in the pipeline transportation. Here flow based algorithm focusing to transport liquid goods with maximum capacity with shortest distance, this algorithm developed with the sense of basic pert and critical path method. Conclusion/Recommendations: This flow based algorithm helps to give optimal result for transporting maximum capacity with minimum cost. It could be used in the juice factory, milk industry and its best alternate for the vehicle routing problem.
\end{abstract}

Key words: Pert, cpm, cyclic, source, destination, routing, transportation

\section{INTRODUCTION}

The shortest-path problem is a classical combinatorial optimization problem in network theory. This problem is to find the shortest route between a source and a destination in a given network. Many complicated problems can be described by it, for instance network routing, (Jigang et al., 2011) vehicle routing, path planning and scheduling. Several good methods have been proposed to solve the problem including the well-known (Lu and Camitz, 2011; Ziliaskopoulos et al., 2009) Dijkstra's algorithm, the Bellman's dynamic programming algorithm, (Mieghem and Fernando, 2004) the Bellman-Ford algorithm and the heuristic algorithm. For the traditional shortest path problem, the objective function is often single, such as minimum cost or minimum distance. In fact, many shortest-path choosing problem in our real life is a shortest path decision problem based on multiobjective, for instance, we might want to find a path for a lorry that minimizes the distance and cost.

Here, it's discussed about construction of pipeline to transport liquid items like petroleum products and liquid items with maximum capacity and with minimum cost through pipe line, this kind of pipe line helps to avoid vehicle routing and its related problem.

Introduction to maximum flow algorithm: The problem is also sometimes called the single-pair shortest path problem, it will help to lay pipe line with shortest distance, to distinguish it from the following
(Ghatee and Hashemi, 2007; Yang and Chen, 2005; Tan, 2001) generalizations:

- The single-source shortest path problem, in which we have to find shortest paths from a source vertex $\mathrm{v}$ to all other vertices in the graph

- The single-destination shortest path problem, in which we have to find shortest paths from all vertices in the graph to a single destination vertex $v$. This can be reduced to the single-source shortest path problem by reversing the edges in the graph

- The all-pairs shortest path problem, in which we have to find shortest paths between every pair of vertices $\mathrm{v}, \mathrm{v}^{\prime}$ in the graph

- These generalizations have significantly more efficient algorithms than the simplistic approach of running a single-pair shortest path algorithm on all relevant pairs of vertices

\section{Introduction to maximum flow algorithm: NOTATIONS:}

Let $\left\{\mathrm{i} \rightarrow \mathrm{j}, \mathrm{D}_{\mathrm{ij}}, \mathrm{W}_{\mathrm{ij}}\right\}$

Where:

$\mathrm{i}=$ Source node

$\mathrm{j}=$ Destination node

$\mathrm{W}_{\mathrm{ij}}=$ Capacity to transport from source $\mathrm{i}$ to destination $\mathrm{j}$

$\mathrm{D}_{\mathrm{ij}}=$ Distance between source $\mathrm{i}$ to destination $\mathrm{j}$

Step 1: Select $\max \left\{i \rightarrow j_{x}, W_{i} j_{x}\right\}$ where $x=123 \ldots n$, by

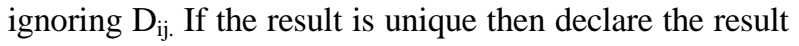
as Initial solution. If not unique then follow step 2 .

Corresponding Author: Sudhakar, V.J., Department of Management Science, Adhiyamaan College of Engineering, Hosur, Tamilnadu 635 109, India 


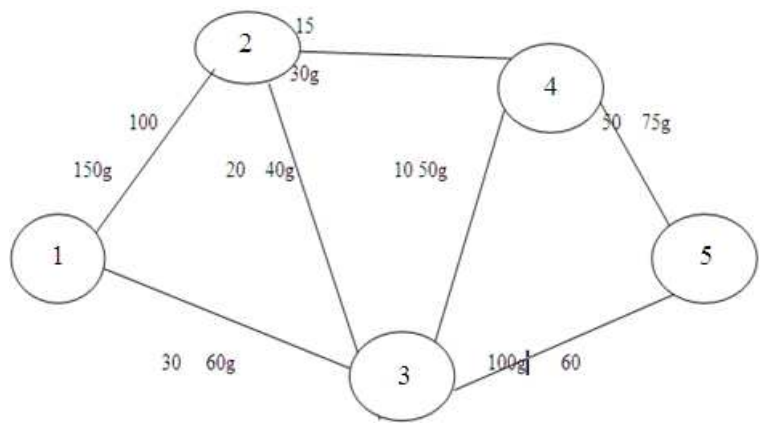

Fig. 1: Eample network with distance and weight

Step 2: If $\operatorname{Max}\left\{\mathrm{i} \rightarrow \mathrm{j}_{\mathrm{x}}, \mathrm{W}_{\mathrm{i}} \mathrm{j}_{\mathrm{x}}\right\}=\left\{\mathrm{Wi} \mathrm{j}_{\mathrm{x}}\right\}$ for $\mathrm{x}=1=2=3 \ldots . .=\mathrm{n}$.

Then select Min $\{$ Dij $\}$ from $\left[\max \left\{i \rightarrow \mathrm{j}_{\mathrm{x}}, \mathrm{W}_{\mathrm{i}} \mathrm{j}_{\mathrm{x}}\right\}\right.$ where $\mathrm{x}=123 \ldots \mathrm{n}]$.

If the result is unique then declare as Initial solution. Once step 2 is over, process must be reverse.

Step 3: Select $\min \left\{j \rightarrow \mathrm{i}_{\mathrm{x}}, \mathrm{D}_{\mathrm{jix}}\right\}$ where $\mathrm{x}=\mathrm{n}$...3.2.1 from the initial solution sequence.

If any contradiction between capacity and distance in the initial solution sequence from $\mathrm{n}$ to 1 , Then select Max ratio $\left\{\mathrm{Wi}_{\mathrm{x}} / \mathrm{Di} \mathrm{j}_{\mathrm{x}}\right\}$ for $\mathrm{x}=\mathrm{n}$.... 1

Step 4: Repeat step 1, 2, 3 till reaching destination node with Minimum distance and Maximum capacity.

Example 1: The following Fig. 1 depicts the pipe line routes to supply liquid goods:

\section{Step 1:}

Select Max $\{[1 \rightarrow 2,150],[1 \rightarrow 3,60]\}$

Stage I solution is $[1 \rightarrow 2,150]$

\section{Step 2:}

Select Max $\{[2 \rightarrow 3,40],[2 \rightarrow 4,30]\}$

Stage II solution is $[2 \rightarrow 3,40]$,

Step 3:

Select Max $\{[3 \rightarrow 4,50],[3 \rightarrow 5,100]\}$

Stage III solution is $[3 \rightarrow 5,100]$

In the above case, in all the stages, solutions are unique and optimal:

$\{1 \rightarrow 2 \rightarrow 3 \rightarrow 5,180,290\}$
Hence the above result is optimal solution for the given situation.

\section{CONCLUSION}

This algorithm gives optimal solution for transporting maximum capacity with shortest route, also its gives idea to lay pipeline in the logistics management also in the network side.

\section{REFERENCES}

Ghatee, M. and S.M. Hashemi, 2007. Descent direction algorithm with multicommodity flow problem for signal optimization and traffic assignment jointly. Applied Math. Comput., 188: 555-566. DOI: 10.1016/j.amc.2006.10.017

Jigang, W., S. Jinb, H. Jib and T. Srikanthanc, 2011. Algorithm for time-dependent shortest safe path on transportation networks. Proc. Comput. Sci., 4: 958-966.

Lu, X. and M. Camitz, 2011. Finding the shortest paths by node combination. Applied Math. Comput., 217: 6401-6408. DOI: 10.1016/j.amc.2011.01.019

Mieghem, P.V. and F.A. Fernando, 2004. Concepts of exact QoS routing algorithms. IEEE/ACM Trans. Network., $\quad 12$ : 851-864. $\quad$ DOI: 10.1109/TNET.2004.836112

Tan, X., 2001. Fast computation of shortest watchman routes in simple polygons. Inform. Process. Lett., 77: 27-33. DOI: 10.1016/S0020-0190(00)00146-0

Yang, H.H. and Y.L. Chen, 2005. Finding $K$ shortest looping paths in a traffic-light network. Comput. Operat. Res., 32: 571-581. DOI: 10.1016/j.cor.2003.08.004

Ziliaskopoulos, A.K., F.D. Mandanas and H.S. Mahmassani, 2009. An extension of labeling techniques for finding shortest path trees. Eur. J. Operat. Res., 198: 63-72. DOI: 10.1016/j.ejor.2008.08.018 